\title{
EXAMPLES OF WEAK HOPF ALGEBRAS ARISING FROM VACANT DOUBLE GROUPOIDS
}

\author{
NICOLÁS ANDRUSKIEWITSCH AND \\ JUAN MARTÍN MOMBELLI
}

\begin{abstract}
We construct explicit examples of weak Hopf algebras (actually face algebras in the sense of Hayashi $[\mathrm{H}]$ ) via vacant double groupoids as explained in [AN]. To this end, we first study the Kac exact sequence for matched pairs of groupoids and show that it can be computed via group cohomology. Then we describe explicit examples of finite vacant double groupoids.
\end{abstract}

\section{Introduction}

Tensor categories have many important applications in several areas of mathematics and theoretical physics. A source of examples of tensor categories is the theory of Hopf algebras; namely the category of representations of a Hopf algebra is naturally a tensor category. However, there are important linear tensor categories that do not arise as the category of representations of any Hopf algebra. Some fifteen years ago, Ocneanu proposed the notion of paragroup to encompass these examples. In this direction, Hayashi introduced face algebras in 1991; eventually, he showed that a suitable linear tensor category arises as the category of representations of a face algebra (canonical Tannaka reconstruction). See $[\mathrm{H}]$ and references therein. Weak Hopf algebras were introduced in [BNS], [BS]; face algebras are weak Hopf algebras with commutative target subalgebra.

Recently, it was explained how to build up weak Hopf algebras (actually face algebras) starting from a matched pairs of finite groupoids and a suitable pair of cocycles [AN]. The purpose of the present paper is to exhibit explicit examples of weak Hopf algebras in the framework of this construction (that we recall in Subsection 2.3). For this, we need to give

Received June 4, 2004.

Revised February 12, 2005.

2000 Mathematics Subject Classification: 17B37; 81R50.

This work was partially supported by Agencia Córdoba Ciencia, ANPCyT-Foncyt, CONICET, ECOS, TWAS (Trieste), Fundación Antorchas and Secyt (UNC). 
explicit examples of matched pairs of finite groupoids (what we do in Section 3), and to compute the corresponding 2 cohomology group (the socalled Opext). As said in [AN], see also [BaSV], an efficient way for this last task is through the Kac exact sequence, a generalization of the analogous sequence for matched pairs of groups. We elaborate on Kac exact sequences in Section 2, relating to cohomology of weak Hopf algebras, which we discuss in Section 1.

The reader interested in the construction of explicit examples might find useful the Summaries 3.3, 3.5, 3.7 and 3.10. We include along the way some calculations of the Opext groups, by reduction to group cohomology.

Notation. We shall denote by $\mathbb{K}$ a commutative ring and by $\mathbb{k}$ a field of characteristic zero. If $R$ is an algebra, we denote by ${ }_{R} \mathcal{M}$ the category of left $R$-modules. If $\mathcal{X}$ is a set, we denote by $\mathbb{K} \mathcal{X}$ the free $\mathbb{K}$-module with basis $(X)_{X \in \mathcal{X}}$. We shall use Sweedler's notation but omitting the summation sign for coalgebras: $\Delta(x)=x_{(1)} \otimes x_{(2)}$, if $\Delta$ is the comultiplication of a coalgebra $C, x \in C$. For any $\operatorname{ring} R$ we shall denote by $R^{\times}$the group of invertible elements in $R$.

Acknowledgments. We thank Sonia Natale for many conversations. Part of the work of the second author was done during a visit to the University of Rheims in the framework of the project ECOS. He is very grateful to Jacques Alev for his kind hospitality.

\section{$\S 1$. Cohomology of groupoids}

\subsection{Weak Hopf algebras}

We first recall the definition of weak Hopf algebras, or quantum groupoids [BNS], [BS]; see also [NV]. Weak Hopf algebras over commutative rings, like groupoid algebras, are considered in [BW]. A weak bialgebra is a collection $(H, m, \Delta)$, where $(H, m)$ is an associative $\mathbb{K}$-algebra with unit 1 and $(H, \Delta)$ is a coassociative $\mathbb{K}$-coalgebra with counit $\varepsilon$, such that the following axioms hold:

$$
\begin{gathered}
\Delta(a b)=\Delta(a) \Delta(b), \quad \forall a, b \in H . \\
\Delta^{(2)}(1)=(\Delta(1) \otimes 1)(1 \otimes \Delta(1))=(1 \otimes \Delta(1))(\Delta(1) \otimes 1) . \\
\varepsilon(a b c)=\varepsilon\left(a b_{(1)}\right) \varepsilon\left(b_{(2)} c\right)=\varepsilon\left(a b_{(2)}\right) \varepsilon\left(b_{(1)} c\right), \quad \forall a, b, c \in H .
\end{gathered}
$$


A weak bialgebra $H$ is a weak Hopf algebra or a quantum groupoid if there exists a linear map $\mathcal{S}: H \rightarrow H$ satisfying

$$
\begin{gathered}
m(\mathrm{id} \otimes \mathcal{S}) \Delta(h)=(\varepsilon \otimes \mathrm{id})(\Delta(1)(h \otimes 1))=: \varepsilon_{t}(h), \\
m(\mathcal{S} \otimes \mathrm{id}) \Delta(h)=(\mathrm{id} \otimes \varepsilon)((1 \otimes h) \Delta(1))=: \varepsilon_{s}(h), \\
m^{(2)}(\mathcal{S} \otimes \mathrm{id} \otimes \mathcal{S}) \Delta^{(2)}=\mathcal{S},
\end{gathered}
$$

for all $h \in H$. The maps $\varepsilon_{s}, \varepsilon_{t}$ are respectively called the source and target maps; their images are called the source and target subalgebras, and we denote them respectively by $H_{s}$ and $H_{t}$.

The weak Hopf algebra $H$ is an $H_{t}$-bimodule via $z . h . w:=z h w$ for $h \in$ $H, z, w \in H_{t}$ and the target subalgebra $H_{t}$ has a left $H$-module structure given by:

$$
h . w=\epsilon_{t}(h w),
$$

for all $h \in H$, and $w \in H_{t}$. This action when restricted to $H_{t}$ gives the left regular action [NV, p. 215]. The following Lemma will be useful later.

Lemma 1.1. Let be $H$ a weak Hopf algebra, $H_{t}$ its target subalgebra, $M$ a left $H_{t}$-module and $N$ a left $H$-module. Then $H \otimes_{H_{t}} M$ has a left $H$-module structure via multiplication on the first tensorand and there are natural isomorphisms

$$
\operatorname{Hom}_{H}\left(H \otimes H_{t} M, N\right) \simeq \operatorname{Hom}_{H_{t}}\left(M, \operatorname{Res}_{H_{t}}^{H} N\right) .
$$

For any projective $H_{t}$-module $M$, the $H$-module $H \otimes_{H_{t}} M$ is projective.

Proof. The desired natural isomorphisms are defined by

$$
\begin{aligned}
& \phi: \operatorname{Hom}_{H}\left(H \otimes H_{t} M, N\right) \rightarrow \operatorname{Hom}_{H_{t}}\left(M, \operatorname{Res}_{H_{t}}^{H} N\right), \phi(f)(m)=f(1 \otimes m), \\
& \psi: \operatorname{Hom}_{H_{t}}\left(M, \operatorname{Res}_{H_{t}}^{H} N\right) \rightarrow \operatorname{Hom}_{H}\left(H \otimes_{H_{t}} M, N\right), \psi(g)(h \otimes m)=h g(m),
\end{aligned}
$$

for all $h \in H, m \in M$. The last claim follows from the first one.

Remark 1.2. If $\mathbb{K}=\mathbb{k}$ is a field then by [NV, Prop. 2.3.4] the target subalgebra is separable and therefore semisimple and thus every $H_{t}$-module is projective. In this case, if $H_{t}$ is commutative then $H$ is a face algebra in the sense of Hayashi [H]. 


\subsection{The Bar resolution for weak Hopf algebras}

Let $H$ be a weak Hopf algebra with target subalgebra $H_{t}$. We define the cohomology groups of $H$ with coefficients in $M \in{ }_{H} \mathcal{M}$ by

$$
\mathrm{H}^{n}(H, M):=\operatorname{Ext}_{H}^{n}\left(H_{t}, M\right)
$$

These cohomology groups can be computed by means of a "normalized bar resolution". Let $\bar{H}$ be the $H_{t}$-bimodule $H / H_{t}$; and let $\bar{h}$ be the class in $\bar{H}$ of $h \in H$. Note that $H \simeq H_{t} \oplus \bar{H}$ as left $H_{t}$-modules, since $H_{t}$ is the image of a projector which is $H_{t}$-linear, cf. [BW, Ch. 6]. Let us assume that $H$ (or equivalently $\bar{H}$ ) is a projective $H_{t}$-module. If $N \in{ }_{H} \mathcal{M}$ and $n \in \mathbb{N}_{0}$, we set

$$
B_{n}(H, N):=H \otimes_{H_{t}} \underbrace{\bar{H} \otimes_{H_{t}} \cdots \otimes_{H_{t}} \bar{H}}_{n \text {-times }} \otimes_{H_{t}} N .
$$

Then $B_{n}(H, N)$ is a left $H$-module via multiplication on the first tensorand, and if $N$ is a projective $H_{t}$-module then $B_{n}(H, N)$ is a projective $H$-module thanks to Lemma 1.1. Let us define maps $\epsilon: B_{0}(H, N) \rightarrow N$, $\partial_{n}: B_{n}(H, N) \rightarrow B_{n-1}(H, N), n>0$, and $s_{n}: B_{n}(H, N) \rightarrow B_{n+1}(H, N)$, $n \geq 0$, by

$$
\begin{gathered}
\epsilon(h \otimes m)=h . m, \\
\partial_{n}\left(h \otimes \overline{h_{1}} \otimes \cdots \otimes \overline{h_{n}} \otimes m\right)=h h_{1} \otimes \overline{h_{2}} \otimes \cdots \otimes \overline{h_{n}} \otimes m \\
+\sum_{i=1}^{n-1}(-1)^{i} h \otimes \overline{h_{1}} \otimes \cdots \otimes \overline{h_{i} h_{i+1}} \otimes \cdots \otimes \overline{h_{n}} \otimes m \\
+(-1)^{n} h \otimes \overline{h_{1}} \otimes \cdots \otimes \overline{h_{n-1}} \otimes h_{n} . m,
\end{gathered}
$$

and

$$
s_{n}\left(h \otimes \overline{h_{1}} \otimes \cdots \otimes \overline{h_{n}} \otimes m\right)=1 \otimes \bar{h} \otimes \overline{h_{1}} \otimes \cdots \otimes \overline{h_{n}} \otimes m,
$$

for all $h, h_{1}, \ldots, h_{n} \in H$ and for all $m \in N$.

LEMma 1.3. For any $n>0$, we have

i) the maps $\partial_{n}$ are well defined $H$-module homomorphisms,

ii) $\partial_{n-1} \partial_{n}=0$, and

iii) $\partial_{n+1} s_{n}+s_{n-1} \partial_{n}=\operatorname{id}_{B_{n}(H, N)}$. 
Proof. We verify i). Assume that $h_{i} \in H_{t}$ for $1<i<n$ then

$$
\begin{aligned}
\partial_{n}\left(h \otimes \overline{h_{1}} \otimes \cdots \otimes \overline{h_{n}} \otimes m\right) \\
=(-1)^{i-1} h \otimes \cdots \otimes \overline{h_{i-1} h_{i}} \otimes \cdots \otimes m \\
\quad+(-1)^{i} h \otimes \cdots \otimes \overline{h_{i} h_{i+1}} \otimes \cdots \otimes m \\
=(-1)^{i-1} h \otimes \cdots \otimes \overline{h_{i-1}} \otimes h_{i} \overline{h_{i+1}} \cdots \otimes m \\
\quad+(-1)^{i} h \otimes \cdots \otimes \overline{h_{i} h_{i+1}} \otimes \cdots \otimes m=0 .
\end{aligned}
$$

The second equality follows since we are taking tensor products over $H_{t}$. For $i=1, n$ the proof is similar. Hence $\partial_{n}$ is well-defined, and it is clearly a $H$-module homomorphism. The proof of ii) is standard and iii) follows by a straightforward calculation.

Lemma 1.3 says that the complex

$$
\begin{aligned}
\cdots & \longrightarrow B_{n}(H, N) \stackrel{\partial_{n}}{\longrightarrow} B_{n-1}(H, N) \longrightarrow \cdots \\
& \longrightarrow B_{2}(H, N) \stackrel{\partial_{2}}{\longrightarrow} B_{1}(H, N) \stackrel{\partial_{1}}{\longrightarrow} B_{0}(H, N) \stackrel{\epsilon}{\longrightarrow} N
\end{aligned}
$$

is acyclic. Thus, we have a projective resolution of the $H$-module $N$ and we can compute the Ext groups $\operatorname{Ext}_{H}^{n}(N, M)$ for any $M \in{ }_{H} \mathcal{M}$, as the cohomology groups $\operatorname{Ext}_{H}^{n}(N, M):=\operatorname{Ker}\left(\partial^{n}\right) / \operatorname{Im}\left(\partial^{n-1}\right)$ of the complex

$$
\begin{aligned}
0 & \longrightarrow C^{0}(N, M) \stackrel{\partial^{0}}{\longrightarrow} C^{1}(N, M) \stackrel{\partial^{2}}{\longrightarrow} \cdots \\
& \longrightarrow C^{n}(N, M) \stackrel{\partial^{n}}{\longrightarrow} C^{n+1}(N, M) \longrightarrow \cdots
\end{aligned}
$$

where $C^{n}(N, M):=\operatorname{Hom}_{H}\left(B_{n}(H, N), M\right)$.

\subsection{Groupoids}

Recall that a (finite) groupoid is a small category (with finitely many arrows), such that every morphism has an inverse. We shall denote a groupoid by $\mathfrak{e}, \mathfrak{s}: \mathcal{G} \rightrightarrows \mathcal{P}$, or simply by $\mathcal{G}$, where $\mathcal{G}$ is the set of arrows, $\mathcal{P}$ is the set of objects and $\mathfrak{e}, \mathfrak{s}$ are the target and source maps. The set of arrows between two objects $P$ and $Q$ is denoted by $\mathcal{G}(P, Q)$ and we shall also denote $\mathcal{G}(P):=\mathcal{G}(P, P)$. The composition map is denoted by $m: \mathcal{G}_{\mathfrak{e}} \times_{\mathfrak{s}} \mathcal{G} \rightarrow \mathcal{G}$, and for two composable arrows $g$ and $h$, that is $\mathfrak{e}(g)=\mathfrak{s}(h)$, the composition will be denoted by juxtaposition: $m(g, h)=g h$.

A morphism between two groupoids is a functor of the underlying categories. If $\mathcal{G} \rightrightarrows \mathcal{P}$, and $\mathcal{H} \rightrightarrows \mathcal{Q}$ are groupoids, and $\phi: \mathcal{G} \rightarrow \mathcal{H}$ is a morphism 
of groupoids, then for any $Q \in \mathcal{P}, \phi\left(\operatorname{id}_{Q}\right)=\operatorname{id}_{\mathfrak{e}\left(\phi\left(\mathrm{id}_{Q}\right)\right)}$, and $\phi$ induces a map $\phi^{0}: \mathcal{P} \rightarrow \mathcal{Q}$, namely $\phi^{0}(Q):=\mathfrak{e}\left(\phi\left(\operatorname{id}_{Q}\right)\right)$. Thus $\mathfrak{e}(\phi(g))=\phi^{0}(\mathfrak{e}(g))$, for any $g \in \mathcal{G}$. If $\phi$ and $\psi$ are two morphisms of groupoids then $(\phi \psi)^{0}=\phi^{0} \psi^{0}$.

We recall a well-known definition.

Definition 1.4. Two morphisms of groupoids $\phi, \psi: \mathcal{G} \rightarrow \mathcal{H}$ are similar, denoted $\phi \sim \psi$, if there is a natural transformation between them; that is, if there exists a map $\tau: \mathcal{P} \rightarrow \mathcal{H}$ such that

$$
\phi(g) \tau(\mathfrak{e}(g))=\tau(\mathfrak{s}(g)) \psi(g), \quad g \in \mathcal{G} .
$$

Observe that "similarity" is an equivalence relation since every natural transformation between two groupoid morphisms is necessarily a natural isomorphism.

Two groupoids $\mathcal{G}, \mathcal{H}$ are similar, and we write $\mathcal{G} \sim \mathcal{H}$, if there is an equivalence of categories between them. In other words, if there are morphisms $\phi: \mathcal{G} \rightarrow \mathcal{H}, \psi: \mathcal{H} \rightarrow \mathcal{G}$ such that $\phi \circ \psi$ and $\psi \circ \phi$ are similar to the corresponding identities.

A basic operation between groupoids is the disjoint union. Namely, if $\mathcal{G} \rightrightarrows \mathcal{P}, \mathcal{H} \rightrightarrows \mathcal{Q}$ are two groupoids, the disjoint union is the groupoid whose set of arrows is $\mathcal{G} \amalg \mathcal{H}$, and whose base is the disjoint union of the bases: $\mathcal{P} \amalg \mathcal{Q}$. If $\mathcal{G} \sim \mathcal{G}^{\prime}$ and $\mathcal{H} \sim \mathcal{H}^{\prime}$ then $\mathcal{G} \amalg \mathcal{G}^{\prime} \sim \mathcal{H} \amalg \mathcal{H}^{\prime}$.

Let us define an equivalence relation on the base $\mathcal{P}$ by $P \approx Q$ if $\mathcal{G}(P, Q) \neq \emptyset$. A groupoid $\mathfrak{e}, \mathfrak{s}: \mathcal{G} \rightrightarrows \mathcal{P}$ is connected if $P \approx Q$ for all $P, Q \in \mathcal{P}$.

Let $S$ be an equivalence class in $\mathcal{P}$ and let $\mathcal{G}_{S}$ denote the corresponding connected groupoid with base $S$; that is, $\mathcal{G}_{S}(P, Q)=\mathcal{G}(P, Q)$ for any $P, Q \in$ $S$. Then the groupoid $\mathcal{G}$ is similar to the disjoint union of the groupoids $\mathcal{G}_{S}: \mathcal{G} \sim \coprod_{S \in \mathcal{P} / \approx \mathcal{G}_{S}}$.

A subgroupoid $\mathcal{H}$ of a groupoid $\mathcal{G}$ is wide if $\mathcal{H}$ has the same base $\mathcal{P}$ as $\mathcal{G}$.

LEMMA 1.5. Let $\mathcal{G}$ be a groupoid.

(i) If $\mathcal{G}$ is connected, then $\mathcal{G} \sim \mathcal{G}(P)$ for any $P \in \mathcal{P}$.

(ii) If $\mathcal{S}$ is a system of representatives of $\mathcal{P} / \approx$ then

$$
\mathcal{G} \sim \coprod_{P \in \mathcal{S}} \mathcal{G}(P)
$$


Proof. (i) Let us fix $P \in \mathcal{P}$. For any $Q \in \mathcal{P}$, denote by $\tau_{Q}$ an element in $\mathcal{G}(P, Q)$ such that $\tau_{P}=\operatorname{id}_{P}$. So, we have defined a map $\tau: \mathcal{P} \rightarrow \mathcal{G}$. Define the following maps $\phi: \mathcal{G} \rightarrow \mathcal{G}(P), \psi: \mathcal{G}(p) \rightarrow \mathcal{G}$ by $\phi(g)=\tau_{\mathfrak{s}(g)} g \tau_{\mathfrak{e}(g)}^{-1}$, $\psi(h)=h$. In fact these maps are morphisms of groupoids. Since we have required that $\tau_{P}=\operatorname{id}_{P}$ then $\phi \circ \psi$ is the identity map. By the definition of $\phi$ we have that $(\psi \circ \phi)(g) \tau_{\mathfrak{e}(g)}=\tau_{\mathfrak{s}(g)} g$, and then $\psi \circ \phi \sim$ id. Part (ii) follows from (i).

Definition 1.6. Given a groupoid $\mathcal{G}$ and a map $p: \mathcal{E} \rightarrow \mathcal{P}$, a left action of $\mathcal{G}$ on $p$ is a map $\triangleright: \mathcal{G}{ }_{\mathfrak{e}} \times p$

$$
p(g \triangleright x)=\mathfrak{s}(g), \quad g \triangleright(h \triangleright x)=g h \triangleright x, \quad \operatorname{id}_{p(x)} \triangleright x=x,
$$

for all composable $g, h \in \mathcal{G}, x \in \mathcal{E}$. We shall say in this case that $(\mathcal{E}, p)$, or $\mathcal{E}$, is a $\mathcal{G}$-bundle.

A right action of $\mathcal{G}$ on $\mathcal{E}$ is a map $\triangleleft: \mathcal{E}_{p} \times_{\mathfrak{s}} \mathcal{G} \rightarrow \mathcal{E}$ such that

$$
p(x \triangleleft g)=\mathfrak{e}(g), \quad(x \triangleright g) \triangleright h=x \triangleleft g h, \quad x \triangleleft \operatorname{id}_{p(x)}=x,
$$

for all composable $g, h \in \mathcal{G}, x \in \mathcal{E}$.

\subsection{The groupoid algebra}

The groupoid algebra $\mathbb{K} \mathcal{G}$ is the $\mathbb{K}$-algebra with basis $\{g: g \in \mathcal{G}\}$, the product of two elements in the basis being equal to their composition if they are composable, and 0 otherwise. The groupoid algebra $\mathbb{K} \mathcal{G}$ has a weak Hopf algebra structure via: $\Delta(g)=g \otimes g, \epsilon(g)=1, S(g)=g^{-1}$, for all $g \in \mathcal{G}$. The target subalgebra of this weak Hopf algebra is $\mathbb{K} \mathcal{P}:=\bigoplus_{P \in \mathcal{P}} \mathbb{K}$ id $P$.

A $\mathcal{G}$-module bundle is a $\mathcal{G}$-bundle $(\mathcal{E}, p)$ such that $\mathcal{E}_{Q}:=p^{-1}(Q)$ is a $\mathbb{K}$-module for any $Q \in \mathcal{P}$ and the map $g \triangleright: \mathcal{E}_{\mathfrak{e}(g)} \rightarrow \mathcal{E}_{\mathfrak{s}(g)}$ is a linear isomorphism for any $g \in \mathcal{G}$.

There is an equivalence of categories between the category of $\mathcal{G}$-module bundles and $\mathbb{K G}_{\mathcal{G}} \mathcal{M}$.

The left $\mathbb{K} \mathcal{G}$-module associated to a $\mathcal{G}$-module bundle $(\mathcal{E}, p)$ is given by $M:=\bigoplus_{Q \in \mathcal{P}} \mathcal{E}_{Q}$, and the action of $\mathcal{G}$ on $M$ is given by $g . m=g \triangleright m$ if $m \in \mathcal{E}_{\mathfrak{e}(g)}$ and $g \cdot m=0$ otherwise. Note that the fiber $\mathcal{E}_{Q}$ might be zero for some $Q \in \mathcal{P}$.

Reciprocally, let $M$ be a left $\mathbb{K} \mathcal{G}$-module and set $M_{P}=\operatorname{id}_{P} M, P \in \mathcal{P}$; then $M=\bigoplus_{P \in \mathcal{P}} M_{P}$. Let

$$
\mathcal{E}:=\left\{(Q, m) \in \mathcal{P} \times M \text { such that } m \in M_{Q}\right\},
$$


let $p: \mathcal{E} \rightarrow \mathcal{P}$ be given by $p(Q, m)=Q$, and let $\triangleright: \mathcal{G}_{\mathfrak{e}} \times{ }_{p} \mathcal{E} \rightarrow \mathcal{E}$ be defined by $g \triangleright(\mathfrak{e}(g), m)=(\mathfrak{s}(g), g \cdot m)$. Then $(\mathcal{E}, p)$ is a $\mathcal{G}$-module bundle.

Proposition 1.7. If $\mathcal{G} \rightrightarrows \mathcal{P}$ and $\mathcal{H} \rightrightarrows \mathcal{Q}$ are similar groupoids then the categories $\mathbb{K} \mathcal{G} \mathcal{M}$ and $\mathbb{k}_{\mathfrak{H}} \mathcal{M}$ are tensor equivalent. In particular the groupoid algebras $\mathbb{K} \mathcal{G}, \mathbb{K} \mathcal{H}$ are Morita equivalent.

Proof. By hypothesis, there are morphisms of groupoids $\phi: \mathcal{G} \rightarrow \mathcal{H}$ and $\psi: \mathcal{H} \rightarrow \mathcal{G}$ satisfying that $\phi \psi \sim \mathrm{id}_{\mathcal{H}}$ and $\psi \phi \sim \mathrm{id}_{\mathcal{G}}$; that is, there are maps $\theta: \mathcal{P} \rightarrow \mathcal{G}$ and $\eta: \mathcal{Q} \rightarrow \mathcal{H}$ such that

$$
\begin{aligned}
\psi \phi(g) \theta(\mathfrak{e}(g)) & =\theta(\mathfrak{s}(g)) g, \\
\phi \psi(h) \eta(\mathfrak{e}(h)) & =\eta(\mathfrak{s}(h)) h,
\end{aligned}
$$

for all $g \in \mathcal{G}$ and $h \in \mathcal{H}$.

We define functors $\Psi: \mathbb{K} \mathcal{G} \mathcal{M} \rightarrow \mathbb{k}_{\mathcal{H}} \mathcal{M}, \Phi: \mathbb{k}_{\mathcal{H}} \mathcal{M} \rightarrow \mathbb{K}_{\mathcal{G}} \mathcal{M}$ by

$$
\Psi(M)_{Q}:=M_{\psi^{0}(Q)}, \quad \text { and } \quad \Phi(V)_{P}:=V_{\phi^{0}(P)},
$$

for all objects $M \in \mathbb{K}_{\mathcal{G}} \mathcal{M}, V \in \mathbb{k}_{\mathcal{H}} \mathcal{M}$ and for all $P \in \mathcal{P}, Q \in \mathcal{Q}$. The action of $\mathcal{H}$ in $\Psi(M)$ is defined as follows: if $x \in \mathcal{H}$ and $m \in \Psi(M)_{Q}$ then

$$
x . m= \begin{cases}\psi(x) m & \text { if } \mathfrak{e}(x)=Q \\ 0 & \text { otherwise }\end{cases}
$$

The action of $\mathcal{G}$ in $\Phi(V)$ is defined as follows: if $g \in \mathcal{G}$ and $v \in \Phi(V)_{P}$ then

$$
g . v= \begin{cases}\phi(g) v & \text { if } \mathfrak{e}(g)=P \\ 0 & \text { otherwise }\end{cases}
$$

Clearly, these are indeed actions of the corresponding groupoids. We define natural isomorphisms $\xi:$ Id $\rightarrow \Psi \Phi$ and $\zeta: \Psi \Phi \rightarrow$ Id by

$$
\begin{aligned}
\xi_{V_{Q}}: V_{Q} \rightarrow V_{(\psi \phi)^{0}(Q)}, \quad \xi_{V_{Q}}(v) & =\theta(Q) v \quad \text { and } \\
\zeta_{M_{P}}: M_{P} \rightarrow M_{(\phi \psi)^{0}(P)}, \quad \zeta_{M_{P}}(m) & =\eta(P) m
\end{aligned}
$$

for all $V \in{ }_{\mathbb{k}} \mathcal{H} \mathcal{M}$ and $M \in \mathbb{K}_{\mathcal{G}} \mathcal{M}$. Equations (1.8), (1.9) imply that $\xi$ and $\zeta$ are morphisms; thus the functors $\Phi$ and $\Psi$ define an equivalence between $\mathbb{K}_{\mathcal{G}} \mathcal{M}$ and ${ }_{\mathbb{k}} \mathcal{H} \mathcal{M}$. A straightforward verification shows that these functors are in fact strict tensor functors. 
Combining Lemma 1.5 and Proposition 1.7 we get

COROLlaRY 1.8. If $\mathcal{G}$ is a connected groupoid then the tensor categories $_{\mathbb{K} \mathcal{G}} \mathcal{M}$ and $\mathbb{k}_{\mathcal{G}(P)} \mathcal{M}$ are tensor equivalent for any $P \in \mathcal{P}$. In particular the groupoid algebra $\mathbb{K} \mathcal{G}$ is Morita equivalent to the group algebra $\mathbb{K} \mathcal{G}(P)$.

\subsection{Groupoid cohomology}

We briefly recall the well-known groupoid cohomology.

Let us fix a groupoid $\mathfrak{e}, \mathfrak{s}: \mathcal{G} \rightrightarrows \mathcal{P}$. Define $\mathcal{G}^{(0)}:=\left\{\operatorname{id}_{Q}\right\}_{Q \in \mathcal{P}}, \mathcal{G}^{(1)}=\mathcal{G}$, and for $n \geq 2$

$$
\mathcal{G}^{(n)}=\left\{\left(g_{1}, \ldots, g_{n}\right) \in \mathcal{G}^{n}: g_{1}\left|g_{2}\right| \cdots\left|g_{n-1}\right| g_{n}\right\} .
$$

Let $(\mathcal{E}, p)$ be a $\mathcal{G}$-module bundle, and define

$$
\begin{aligned}
& C^{0}(\mathcal{G}, \mathcal{E})=\{f: \mathcal{P} \rightarrow \mathcal{E}: p(f(Q))=Q \forall Q \in \mathcal{P}\} \\
& C^{n}(\mathcal{G}, \mathcal{E})=\left\{f: \mathcal{G}^{(n)} \rightarrow \mathcal{E}: f\left(g_{1}, \ldots, g_{n}\right)=0, \text { if some } g_{i} \in \mathcal{G}^{(0)},\right. \\
&\left.\quad \text { and } p\left(f\left(g_{1}, \ldots, g_{n}\right)\right)=\mathfrak{s}\left(g_{1}\right) \forall\left(g_{1}, \ldots, g_{n}\right) \in \mathcal{G}^{(n)}\right\} .
\end{aligned}
$$

The cohomology groups $H^{n}(\mathcal{G}, \mathcal{E})$ of $\mathcal{G}$ with coefficients in the $\mathcal{G}$-module bundle $(\mathcal{E}, p)$ are the cohomology groups of the complex

$$
\begin{aligned}
0 \longrightarrow C^{0}(\mathcal{G}, \mathcal{E}) \stackrel{d_{0}}{\longrightarrow} C^{1}(\mathcal{G}, \mathcal{E}) \stackrel{d_{1}}{\longrightarrow} C^{2}(\mathcal{G}, \mathcal{E}) \stackrel{d_{2}}{\longrightarrow} \cdots \\
\longrightarrow C^{n}(\mathcal{G}, \mathcal{E}) \stackrel{d_{n}}{\longrightarrow} C^{n+1}(\mathcal{G}, \mathcal{E}) \longrightarrow \cdots
\end{aligned}
$$

where

$$
\begin{aligned}
d^{n} f\left(g_{0}, \ldots, g_{n}\right)=g_{0} \triangleright & f\left(g_{1}, \ldots, g_{n}\right)+\sum_{i=1}^{n}(-1)^{i} f\left(g_{0}, \ldots, g_{i-1} g_{i}, \ldots, g_{n}\right) \\
+ & (-1)^{n+1} f\left(g_{0}, \ldots, g_{n-1}\right) .
\end{aligned}
$$

Let us denote as usual $Z^{n}(\mathcal{G}, M):=\operatorname{Ker}\left(d^{n}\right), B^{n}(\mathcal{G}, M):=\operatorname{Im}\left(d^{n-1}\right)$, $n \geq 0$. We next show that this groupoid cohomology coincides with the cohomology of the weak Hopf algebra $\mathbb{K} \mathcal{G}$.

Proposition 1.9. If $\mathcal{E}$ is a $\mathcal{G}$-module bundle and $M$ is the associated $\mathbb{K} \mathcal{G}$-module, then the groups $H^{n}(\mathcal{G}, \mathcal{E})$ and $H^{n}(\mathbb{K} \mathcal{G}, M)$ are naturally isomorphic. 
Proof. Let $C^{n}(\mathbb{K} \mathcal{P}, M):=\operatorname{Hom}_{\mathbb{K} \mathcal{G}}\left(B_{n}(\mathbb{K} \mathcal{G}, \mathbb{K} \mathcal{P}), M\right)$ as in Section 1.2. Let us define $F_{n}: C^{n}(\mathbb{K} \mathcal{P}, M) \rightarrow C^{n}(\mathcal{G}, \mathcal{E})$ by

$$
\begin{aligned}
F_{0}(f)(P) & =f\left(\operatorname{id}_{P}\right), & P & \in \mathcal{P}, \\
F_{n}(f)\left(g_{1}, \ldots, g_{n}\right) & =f\left(\operatorname{id}_{\mathfrak{s}\left(g_{1}\right)} \otimes \overline{g_{1}} \otimes \cdots \otimes \overline{g_{n}}\right), & \left(g_{1}, \ldots, g_{n}\right) & \in \mathcal{G}^{(n)} .
\end{aligned}
$$

Then $F_{n}$ are isomorphisms whose inverses are the maps $G_{n}: C^{n}(\mathcal{G}, \mathcal{E}) \rightarrow$ $C^{n}(\mathbb{K} \mathcal{P}, M)$ given by

$$
\begin{aligned}
G_{0}(f)(g) & =g \triangleright f(\mathfrak{e}(g)), \\
G_{n}(f)\left(g_{o} \otimes \overline{g_{1}} \otimes \cdots \otimes \overline{g_{n}}\right) & =g_{0} \triangleright f\left(g_{1}, \ldots, g_{n}\right) .
\end{aligned}
$$

It follows from the definition of the maps $d^{n}$ that $d^{n} F_{n}=F_{n} \partial^{n}$ for any $n \geq 0$. Thus, the maps $F_{n}$ induce isomorphisms $H^{n}(\mathcal{G}, \mathcal{E}) \rightarrow H^{n}(\mathbb{K} \mathcal{G}, M)$.

As a consequence, we show that groupoid cohomology can be derived from group cohomology.

Proposition 1.10. (i) Let $\mathcal{E}$ be a $\mathcal{G}$-module bundle and let $S$ be a complete set of representatives of equivalence classes in $\mathcal{P}$. Then there are natural isomorphisms-induced by the respective inclusions

$$
H^{n}(\mathcal{G}, \mathcal{E}) \simeq \bigoplus_{P \in S} H^{n}\left(\mathcal{G}(P), \mathcal{E}_{P}\right)
$$

(ii) Assume that $\mathcal{G}$ is connected and let us fix $O \in \mathcal{P}$. Let $\mathcal{E}$ be a $\mathcal{G}$ module bundle and let $\mathcal{H}$ be a connected wide subgroupoid of $\mathcal{G}$. Set $G=$ $\mathcal{G}(O), H=\mathcal{H}(O)$. Then the following diagram commutes:

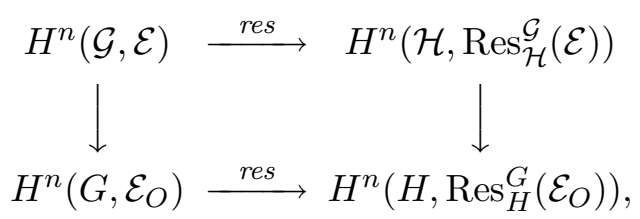

where the vertical arrows are the isomorphisms from part (i).

Proof. (i) Combine (1.7), Proposition 1.9, and the fact that the Ext groups are Morita invariant. Here the well-known natural isomorphisms $\operatorname{Ext}_{A \times B}^{n}(M \oplus N, U \oplus V) \simeq \operatorname{Ext}_{A}^{n}(M, U) \oplus \operatorname{Ext}_{B}^{n}(N, V), n \in \mathbb{N}$, are present, where $A$ and $B$ are rings, $M$ and $U$ are $A$-modules and $N$ and $V$ are $B$ modules.

(ii) Straightforward. 
Definition 1.11. Let $A$ be a $\mathbb{K}$-module. We shall denote by $\underline{A}$ the $\mathcal{G}$-module bundle such that $\underline{A}_{P}:=A, P \in \mathcal{P}$, with trivial action of $\mathcal{G}$. That is, $\underline{A}$ is the $\mathcal{G}$-module bundle corresponding to the $\mathbb{K} \mathcal{G}$-module $\mathbb{K} \mathcal{P} \otimes_{\mathbb{K}} A$. By Proposition $1.10(\mathrm{i}), H^{n}(\mathcal{G}, \underline{A}) \simeq \bigoplus_{P \in S} H^{n}(\mathcal{G}(P), A)$.

Observe that if $A$ is a $\mathbb{K}$-module then the set $Z^{2}(\mathcal{G}, \underline{A})$ is identified with the set of maps $\sigma: \mathcal{G}_{\mathfrak{e}} \times{ }_{\mathfrak{s}} \mathcal{G} \rightarrow A$ such that

$$
\sigma(g, h f) \sigma(h, f)=\sigma(g h, f) \sigma(g, h),
$$

for composable $g, h, f \in \mathcal{G}$.

\section{§2. The Kac exact sequence for matched pairs of groupoids}

\subsection{Matched pairs of groupoids}

We briefly recall the definition of matched pair of groupoids, and the equivalent formulations in terms of exact factorizations or vacant double groupoids, see $[\mathrm{Ma}]$ or $[\mathrm{AN}]$ for details.

A matched pair of groupoids is a collection $(\mathcal{H}, \mathcal{V}, \triangleright, \triangleleft)$, where $b, t: \mathcal{V} \rightrightarrows$ $\mathcal{P}$ and $r, l: \mathcal{H} \rightrightarrows \mathcal{P}$ are two groupoids over the same base $\mathcal{P}, \triangleright: \mathcal{H}_{r} \times_{t} \mathcal{V} \rightarrow \mathcal{V}$ is a left action of $\mathcal{H}$ on $(\mathcal{V}, t), \triangleleft: \mathcal{H}_{r} \times_{t} \mathcal{V} \rightarrow \mathcal{H}$ is a right action of $\mathcal{V}$ on $(\mathcal{H}, r)$ such that

$$
\begin{gathered}
b(x \triangleright g)=l(x \triangleleft g), \\
x \triangleright g h=(x \triangleright g)((x \triangleleft g) \triangleright h), \\
x y \triangleleft g=(x \triangleleft(y \triangleright g))(y \triangleleft g),
\end{gathered}
$$

for composable elements $x, y \in \mathcal{H}$ and $g, h \in \mathcal{V}$. Here and below we use the 'horizontal and vertical notation': the source and target of $\mathcal{H}$, resp. $\mathcal{V}$, are denoted $l$ and $r$ (left and right), resp. $t$ and $b$ (top and bottom).

Let $(\mathcal{H}, \mathcal{V}, \triangleleft, \triangleright)$ be a matched pair of groupoids. There is an associated diagonal groupoid $\mathcal{V} \bowtie \mathcal{H}$ with set of arrows $\mathcal{V}_{b} \times_{l} \mathcal{H}$, base $\mathcal{P}$, source, target, composition and identity given by

$$
\begin{gathered}
\mathfrak{s}(g, x)=t(g), \quad \mathfrak{e}(g, x)=r(x), \\
(g, x)(h, y)=(g(x \triangleright h),(x \triangleleft h) y), \quad \operatorname{id}_{P}=\left(\operatorname{id}_{P}, \operatorname{id}_{P}\right),
\end{gathered}
$$

$g, h \in \mathcal{V}, x, y \in \mathcal{H}, P \in \mathcal{P}$. Then we have an exact factorization of groupoids $\mathcal{V} \bowtie \mathcal{H}=\mathcal{V H}$. Conversely, if $\mathcal{D}=\mathcal{V H}$ is an exact factorization of groupoids 
then there are actions $\triangleleft, \triangleright$ such that $(\mathcal{H}, \mathcal{V}, \triangleleft, \triangleright)$ form a matched pair of groupoids, and $\mathcal{D} \simeq \mathcal{V} \bowtie \mathcal{H}$.

There is also a vacant double groupoid associated to the matched pair of $\mathcal{B} \rightrightarrows \mathcal{H}$ groupoids $(\mathcal{H}, \mathcal{V})$. In simple terms, this is a collection of groupoids $\mathcal{V} \rightrightarrows \mathcal{P}$ with the following meaning. A pair $(x, g)$ in $\mathcal{B}:=\mathcal{H}_{r} \times_{t} \mathcal{V}$ is depicted as a box $h \square_{y}^{x} g$ where $h=x \triangleright g, y=x \triangleleft g$. The horizontal groupoid $r, l: \mathcal{B} \rightrightarrows \mathcal{V}$ has source, target, composition and identity given by

$$
r(x, g)=g, \quad l(x, g)=x \triangleright g, \quad(x, g)(y, h)=(x y, h), \quad \operatorname{id}(g)=\left(\operatorname{id}_{t(g)}, g\right)
$$

$x, y \in \mathcal{H}, g, h \in \mathcal{V}$. The vertical groupoid $t, b: \mathcal{B} \rightrightarrows \mathcal{H}$ has source, target, composition and identity given by

$$
b(x, g)=x \triangleleft g, \quad t(x, g)=x, \quad(x, g)(y, h)=(x, g h), \quad \operatorname{id}(x)=\left(x, \operatorname{id}_{r(x)}\right)
$$

$x, y \in \mathcal{H}, g, h \in \mathcal{V}$. If $A, B$ are two boxes we denote $A \mid B$ if they are horizontally composable, and $\frac{A}{B}$ if they are vertically composable, that is if $A=h \square_{y}^{x} g$ and $B=f \underset{w}{\square_{w}} k$, then $A \mid B$ if and only if $g=f$, and $\frac{A}{B}$ if and only if $y=z$.

\subsection{The Kac exact sequence}

In this Subsection we shall review and complete details of the proof of the Kac exact sequence for vacant double groupoids introduced in [AN]. Let $(\mathcal{H}, \mathcal{V}, \triangleleft, \triangleright)$ be a matched pair of groupoids. We begin by a non standard resolution of the diagonal groupoid, adapting ideas from [M1] to the groupoid case. If $r, s \in \mathbb{N}$, we denote by $\mathcal{B}^{[r, s]}$ the set of matrices

$$
\left(\begin{array}{cccc}
A_{11} & A_{12} & \cdots & A_{1 s} \\
A_{21} & A_{22} & \cdots & A_{2 s} \\
\cdots & \cdots & \cdots & \cdots \\
A_{r 1} & A_{r 2} & \cdots & A_{r s}
\end{array}\right) \in \mathcal{B}^{r \times s}
$$

such that 
- For all $i, j, A_{i j} \mid A_{i, j+1}, \frac{A_{i j}}{A_{i+1, j}}$. This condition is summarized in the notation:

\begin{tabular}{l|l|l|l}
$A_{11}$ & $A_{12}$ & $\cdots$ & $A_{1 s}$ \\
\hline$A_{21}$ & $A_{22}$ & $\cdots$ & $A_{2 s}$ \\
\hline$\cdots$ & $\cdots$ & $\cdots$ & $\cdots$ \\
\hline$A_{r 1}$ & $A_{r 2}$ & $\cdots$ & $A_{r s}$
\end{tabular}

- If $j<s$ then $A_{i j}$ is not a horizontal identity.

- If $i>1$ then $A_{i j}$ is not a vertical identity.

Observe that if $A=\left(A_{i j}\right)$ is an element in $\mathcal{B}^{[r, s]}$ then $A$ is determined by $s$ composable elements $x_{1}, \ldots, x_{s}$ in $\mathcal{H}$-those in the top of the arrayand $r$ composable elements $g_{1}, \ldots, g_{r}$ in $\mathcal{V}$-those in the right side of the array, with $r\left(x_{s}\right)=t\left(g_{1}\right)$. We shall denote it by

$$
A=: \stackrel{x_{1}, \ldots, x_{s}}{\square} g_{1}, \ldots, g_{r}
$$

Let $s_{V}^{r, s}: \mathbb{K} \mathcal{B}^{[r, s]} \rightarrow \mathbb{K}^{[r+1, s]}$ (vertical homotopy maps), $\partial_{V}^{r, s}: \mathbb{K}^{[r, s]} \rightarrow$ $\mathbb{K} \mathcal{B}^{[r-1, s]}$ and $\partial_{H}^{r, s}: \mathbb{K} \mathcal{B}^{[r, s]} \rightarrow \mathbb{K}^{[r, s-1]}$ (vertical and horizontal coboundary maps) be defined by

$$
\begin{aligned}
& s_{V}^{r, s}\left(x_{1}^{x_{1}, \ldots, x_{s}} g_{1}, \ldots, g_{r}\right):=\square \operatorname{li}_{t\left(g_{1}\right)}, g_{1}, \ldots, g_{r}
\end{aligned}
$$

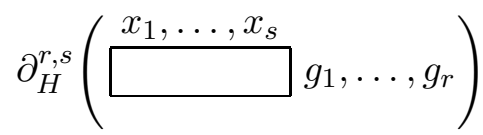

$$
\begin{aligned}
& =\sum_{j=1}^{s-1}(-1)^{s-j-1} \stackrel{x_{1}, \ldots, x_{j} x_{j+1}, \ldots, x_{s}}{\square} g_{1}, \ldots, g_{r} \\
& +(-1)^{s-1} \stackrel{x_{2}, \ldots, x_{s}}{\square} g_{1}, \ldots, g_{r}
\end{aligned}
$$




$$
\begin{gathered}
\partial_{V}^{r, s}\left(\begin{array}{l}
x_{1}, \ldots, x_{s} \\
\square
\end{array} g_{1}, \ldots, g_{r}\right) \\
=\sum_{i=1}^{r-1}(-1)^{i-1} g_{1}, \ldots, g_{i} g_{i+1}, \ldots, g_{r} \\
\quad+(-1)^{r-1} x_{1}, \ldots, x_{s} \\
\end{gathered}
$$

A straightforward computation shows that the following diagram commutes:

$$
\begin{array}{crc}
\mathbb{K} \mathcal{B}^{[r, s]} & \stackrel{\partial_{H}^{r, s}}{\longrightarrow} & \mathbb{K} \mathcal{B}^{[r, s-1]} \\
\partial_{V}^{r, s} \downarrow & \downarrow \partial_{V}^{r, s-1} \\
\mathbb{K} \mathcal{B}^{[r-1, s]} & \stackrel{\partial_{H}^{r-1, s}}{\longrightarrow} & \mathbb{K} \mathcal{B}^{[r-1, s-1]} .
\end{array}
$$

Thus, we have constructed a double chain complex $\mathfrak{B}^{\bullet} \bullet$ :

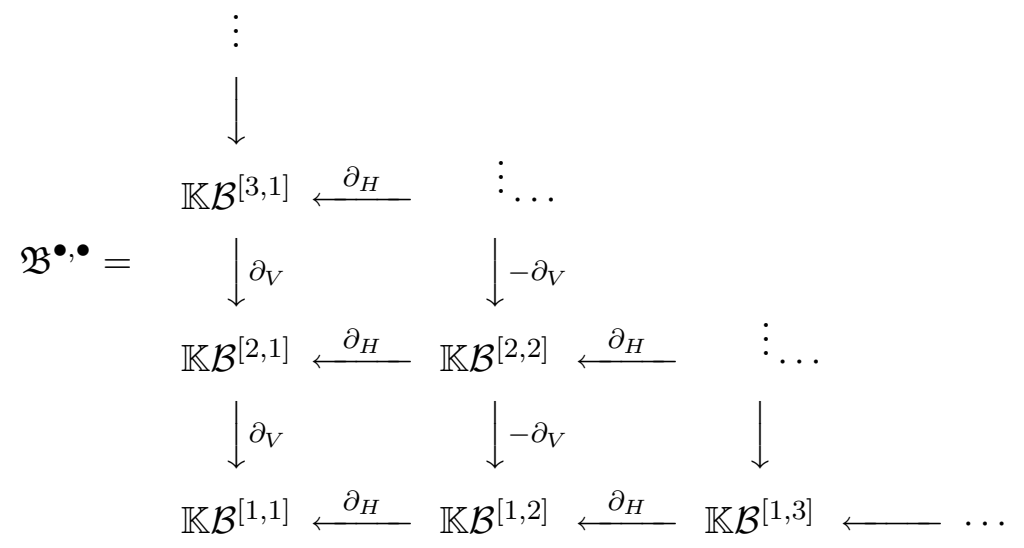

Note that $\mathcal{B}^{[r, s]}$ is a subset of the set $\mathcal{B}^{(r, s)}$ defined in $[\mathrm{AN}]$; and the double complex presented here is different from the double complex in [AN]. However these will be reconciliated in Remark 2.5 below.

Let us now define an action of the diagonal groupoid $\mathcal{V} \bowtie \mathcal{H}$ on $\mathbb{K} \mathcal{B}^{[r, s]}$, $r, s>0$. The $\mathcal{P}$-gradation on $\mathbb{K} \mathcal{B}^{[r, s]}$ is given by:

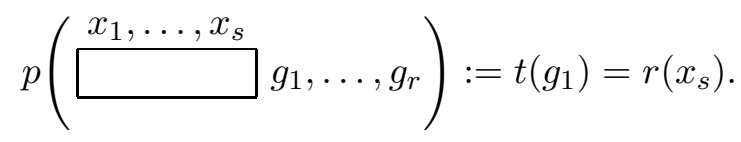


If $h \in \mathcal{V}, y \in \mathcal{H}$ are such that $r(y)=r\left(x_{s}\right)=t\left(g_{1}\right)=b(h)$, then we set

$$
\begin{aligned}
& y \cdot\left(x_{1}, \ldots, x_{s}\right. \\
& \left.g_{1}, \ldots, g_{r}\right) \\
& \quad:=\square y \triangleright g_{1},\left(y \triangleleft g_{1}\right) \triangleright g_{2}, \ldots,\left(y \triangleleft g_{1} \ldots g_{r-1}\right) \triangleright g_{r},
\end{aligned}
$$

$$
\begin{aligned}
& h \cdot\left(x_{1}^{x_{1}, \ldots, x_{s}} g_{1}, \ldots, g_{r}\right) \\
& \quad:=x_{1} \triangleleft\left(x_{2} \ldots x_{s} \triangleright h^{-1}\right), \ldots, x_{s} \triangleleft h^{-1} \\
&
\end{aligned}
$$

LEMMA 2.1. (i) The rules (2.2) and (2.3) induce a structure of $\mathbb{K} \mathcal{V} \bowtie \mathcal{H}$-module on $\mathbb{K} \mathcal{B}^{[r, s]}$.

(ii) There are $\mathbb{K} \mathcal{V} \bowtie \mathcal{H}$-isomorphisms:

- $\mathbb{K} \mathcal{B}^{[r, s]} \simeq \mathbb{K} \mathcal{V} \bowtie \mathcal{H} \otimes_{\mathbb{K} \mathcal{P}} \mathbb{K} \mathcal{B}^{[r-1, s-1]}$ for any $r, s>1$,

- $\mathbb{K} \mathcal{B}^{[r, 1]} \simeq \mathbb{K} \mathcal{V} \bowtie \mathcal{H} \otimes_{\mathbb{K} \mathcal{P}} \mathbb{K} \mathcal{V}^{(r-1)}$ for any $r>0$,

- $\mathbb{K} \mathcal{B}^{[1, s]} \simeq \mathbb{K} \mathcal{V} \bowtie \mathcal{H} \otimes_{\mathbb{K} \mathcal{P}} \mathbb{K} \mathcal{H}^{(s-1)}$ for any $s>0$,

where the action of $\mathbb{K} \mathcal{V} \bowtie \mathcal{H}$ on $\mathbb{K} \mathcal{V} \bowtie \mathcal{H} \otimes_{\mathbb{K} \mathcal{P}} L$ is on the first tensorand, for any $L \in \mathbb{K} \mathcal{P} \mathcal{M}$.

(iii) $\mathbb{K B}^{[r, s]}$ is a projective $\mathbb{K} \mathcal{V} \bowtie \mathcal{H}$-module for any $r, s>0$.

(iv) The coboundary maps $\partial_{V}^{r, s}, \partial_{H}^{r, s}$ are morphisms of $\mathbb{K} \mathcal{V} \bowtie \mathcal{H}$-modules.

(v) $\partial_{V}^{r+1, s} s_{V}^{r, s}+s_{V}^{r-1, s} \partial_{V}^{r, s}=\operatorname{id}_{\mathbb{K} \mathcal{B}[r, s]}$.

Proof. (i) Let $A=x^{x_{1}, \ldots, x_{s}} g_{1}, \ldots, g_{r} \in \mathcal{B}^{[r, s]}$; let $h \in \mathcal{V}, y \in \mathcal{H}$,

such that $r(y)=t(h)$ and $b(h)=p(A)$. We claim that $y \cdot(h . A)=(y \triangleright h) .((y \triangleleft$ h).A). We have

$$
y .(h . A)=\square y \triangleright h g_{1},\left(y \triangleleft h g_{1}\right) \triangleright g_{2}, \ldots,\left(y \triangleleft h g_{1} \ldots g_{r-1}\right) \triangleright g_{r} ;
$$


and $(y \triangleright h) \cdot((y \triangleleft h) \cdot A)=$ $x_{1} \triangleleft\left(\left(x_{2} \ldots x_{s}(y \triangleleft h)^{-1}\right) \triangleright(y \triangleright h)^{-1}\right), \ldots,\left(x_{s}(y \triangleleft h)^{-1}\right) \triangleleft(y \triangleright h)^{-1}(y \triangleright h)\left((y \triangleleft h) \triangleright g_{1}\right), \ldots,\left(\left(y \triangleleft h g_{1} \ldots g_{r-1}\right) \triangleright g_{r}\right.$.

Then $y \cdot(h . A)=(y \triangleright h) \cdot((y \triangleleft h) . A)$ by $(2.1)$ and the identities

$$
(y \triangleright h)^{-1}=(y \triangleleft h) \triangleright h^{-1}, \quad(y \triangleleft h)^{-1}=y^{-1} \triangleleft(y \triangleright h) .
$$

(ii) Assume that $r, s>1$. Define the maps $\phi: \mathbb{K} \mathcal{B}^{[r, s]} \rightarrow \mathbb{K} \mathcal{V} \bowtie \mathcal{H} \otimes_{\mathbb{K} \mathcal{P}}$ $\mathbb{K} \mathcal{B}^{[r-1, s-1]}, \psi: \mathbb{K} \mathcal{V} \bowtie \mathcal{H} \otimes_{\mathbb{K} \mathcal{P}} \mathbb{K} \mathcal{B}^{[r-1, s-1]} \rightarrow \mathbb{K} \mathcal{B}^{[r, s]}$ by the formulas

$$
\begin{gathered}
\phi\left(\begin{array}{c}
x_{1}, \ldots, x_{s} \\
\square
\end{array} g_{1}, \ldots, g_{r}\right):=\left(x_{s}^{-1}, x_{s} \triangleright g_{1}\right) \\
\otimes \square\left(x_{1} \triangleleft\left(x_{2} \ldots x_{s} \triangleright g_{1}\right), \ldots, x_{s-1} \triangleleft\left(x_{s} \triangleright g_{1}\right)\right. \\
\end{gathered}
$$

$$
\begin{aligned}
& \psi\left((y, h) \otimes \square g_{1}, \ldots, g_{r-1}\right) \\
& \quad:=(y, h) \cdot\left(x_{1}, \ldots, x_{s-1}, \operatorname{id}_{r\left(x_{s-1}\right)} \operatorname{id}_{t\left(g_{1}\right)}, g_{1}, \ldots, g_{r-1}\right) .
\end{aligned}
$$

These maps are morphisms of $\mathbb{K} \mathcal{V} \bowtie \mathcal{H}$-modules and one is the inverse of each other. The proof of the cases $r=1$ or $s=1$ follows similarly. Part (iii) follows from (ii) and Lemma 1.1. The proof of (iv) and (v) is straightforward.

Let $\mathcal{A}^{\bullet \bullet \bullet}$ be the double chain complex obtained from $\mathfrak{B}^{\bullet \bullet \bullet}$ by removing the edges; that is $\mathcal{A}^{r, s}:=\mathfrak{B}^{r+1, s+1}$. Let $M$ be a left $\mathbb{K} \mathcal{V} \bowtie \mathcal{H}$-module and thus a left $\mathbb{K} \mathcal{H}$-module and also a left $\mathbb{K} \mathcal{V}$-module. Define the double cochain complexes $\mathfrak{B}^{\bullet \bullet \bullet}(M), \mathcal{E}^{\bullet \bullet \bullet}(M), \mathcal{A}^{\bullet \bullet \bullet}(M)$ by

$$
\begin{aligned}
\mathfrak{B}^{r, s}(M) & :=\operatorname{Hom}_{\mathbb{K} \mathcal{V} \bowtie \mathcal{H}}\left(\mathbb{K} \mathcal{B}^{[r, s]}, M\right), \\
\mathcal{A}^{r, s}(M) & :=\operatorname{Hom}_{\mathbb{K} \mathcal{V} \bowtie \mathcal{H}}\left(\mathbb{K}^{[r+1, s+1]}, M\right),
\end{aligned}
$$

and $\mathcal{E}^{r, s}(M)$ consists only of the edges of $\mathfrak{B}(M)$. 
Remark 2.2. Let $M$ be a $\mathbb{K} \mathcal{V} \bowtie \mathcal{H}$-module. Lemma 2.1 (ii) implies that there are natural $\mathbb{K}$-linear isomorphisms: $\mathfrak{B}^{r, s}(M) \simeq \operatorname{Hom}_{\mathbb{K}}\left(\mathbb{K} \mathcal{B}^{[r-1, s-1]}, M\right)$ for any $r, s>1$, and there are natural bijections: $\mathfrak{B}^{r, 1}(M) \simeq C^{(r-1)}(\mathcal{V}, \mathcal{E})$, $\mathfrak{B}^{1, s}(M) \simeq C^{(s-1)}(\mathcal{H}, \mathcal{E})$ for any $r, s>0$, where $\mathcal{E}$ is the module bundle corresponding to $M$.

Remark 2.3. Let $\mathcal{B}^{(r, s)}$ be as in [AN]. Suppose that $r, s>1$. We extend any $\mu \in \mathfrak{B}^{r, s}(M) \simeq \operatorname{Hom}_{\mathbb{K}}\left(\mathbb{K} \mathcal{B}^{[r-1, s-1]}, M\right)$ to $\widetilde{\mu} \in \operatorname{Hom}_{\mathbb{K}}\left(\mathbb{K} \mathcal{B}^{(r-1, s-1)}, M\right)$ by 0 on $\mathcal{B}^{(r-1, s-1)}-\mathcal{B}^{[r-1, s-1]}$. In other words, the elements of $\mathfrak{B}^{r, s}(M)$ are normalized by definition.

Now we can formulate the Kac exact sequence for groupoids.

Theorem 2.4. ([AN, Prop. 3.14]) Let $M$ be a $\mathbb{K} \mathcal{V} \bowtie \mathcal{H}$-module. Then, there is an exact sequence

$$
\begin{aligned}
0 & \longrightarrow H^{1}(\mathcal{D}, M) \stackrel{\text { res }}{\longrightarrow} H^{1}(\mathcal{H}, M) \oplus H^{1}(\mathcal{V}, M) \longrightarrow H^{0}\left(\operatorname{Tot} \mathcal{A}^{\bullet \bullet \bullet}(M)\right) \\
& \longrightarrow H^{2}(\mathcal{D}, M) \stackrel{\text { res }}{\longrightarrow} H^{2}(\mathcal{H}, M) \oplus H^{2}(\mathcal{V}, M) \longrightarrow H^{1}\left(\operatorname{Tot} \mathcal{A}^{\bullet \bullet \bullet}(M)\right) \\
& \longrightarrow H^{3}(\mathcal{D}, M) \stackrel{\text { res }}{\longrightarrow} H^{3}(\mathcal{H}, M) \oplus H^{3}(\mathcal{V}, M) \longrightarrow \cdots
\end{aligned}
$$

The maps denoted by res in the above exact sequence come from the usual restriction maps.

Proof. The short exact sequence of double complexes $0 \rightarrow \mathcal{A}^{\bullet \bullet \bullet}(M) \rightarrow$ $\mathfrak{B}^{\bullet \bullet \bullet}(M) \rightarrow \mathcal{E}^{\bullet \bullet \bullet}(M) \rightarrow 0$ induces a long exact sequence in cohomology. By Remark 2.2 it is easy to see that

$$
H^{n}\left(\operatorname{Tot}^{\bullet}, \bullet(M)\right) \simeq H^{n}(\mathcal{H}, M) \oplus H^{n}(\mathcal{V}, M)
$$

for any $n \in \mathbb{N}_{0}$. By Lemma 2.1 (v) each column of $\mathfrak{B}^{\bullet \bullet \bullet}$ is acyclic. Hence the associated total complex

$$
\begin{aligned}
\cdots & \longrightarrow \operatorname{Tot}(\mathfrak{B})_{n} \stackrel{\partial_{n}}{\longrightarrow} \operatorname{Tot}(\mathfrak{B})_{n-1} \longrightarrow \cdots \\
& \longrightarrow \operatorname{Tot}(\mathfrak{B})_{3} \stackrel{\partial_{1}}{\longrightarrow} \operatorname{Tot}(\mathfrak{B})_{2} \stackrel{\epsilon}{\longrightarrow} \mathbb{K} \mathcal{P},
\end{aligned}
$$

where $\epsilon: \operatorname{Tot}(\mathfrak{B})_{1} \rightarrow \mathbb{K} \mathcal{P}$ is given by the degree: $\epsilon(\square g)=l(x)$, is a projective resolution of the trivial $\mathbb{K} \mathcal{V} \bowtie \mathcal{H}$-module. See, for instance, [W, Ex. 1.2.5]. Hence $H^{n}(\operatorname{Tot} \mathfrak{B}(M)) \simeq H^{n}(\mathcal{V} \bowtie \mathcal{H}, M)$ for any $n \in \mathbb{N}_{0}$, and this finishes the proof. 
Remark 2.5. Let now $\mathbb{K}=\mathbb{Z}$ and $A=\mathbb{k}^{\times}$and recall the meaning of $\underline{\mathbb{k}^{\times}}$in Definition 1.11. Let us denote

$$
\operatorname{Aut}(\mathbb{k} \mathcal{T}):=H^{0}\left(\operatorname{Tot} \mathcal{A}^{\bullet, \bullet}\left(\underline{\mathbb{k}^{\times}}\right)\right), \quad \operatorname{Opext}(\mathcal{V}, \mathcal{H}):=H^{1}\left(\operatorname{Tot} \mathcal{A}^{\bullet}, \bullet\left(\underline{\mathbb{k}^{\times}}\right)\right) .
$$

The group $Z^{2}\left(\operatorname{Tot} \mathcal{A}^{\bullet}, \bullet\left(\underline{\mathbb{k}^{\times}}\right)\right)$can be identified with set of pairs $(\sigma, \tau)$ such that $\sigma$ is a normalized 2-cocycle with values in $\underline{\mathbb{k}^{\times}}$for the vertical groupoid $\mathcal{B} \rightrightarrows \mathcal{H}, \tau$ is a normalized 2 -cocycle with values in $\underline{\mathbb{k}^{\times}}$for the horizontal groupoid $\mathcal{B} \rightrightarrows \mathcal{V}$ and

$$
\sigma(A B, C D) \tau\left(\begin{array}{ll}
A & B \\
C^{\prime} & D
\end{array}\right)=\tau(A, B) \tau(C, D) \sigma(A, C) \sigma(B, D)
$$

for any $A, B, C, D$ such that $\frac{A}{C} \mid$\begin{tabular}{l}
$D$ \\
\hline$C$
\end{tabular} . Hence, $\operatorname{Opext}(\mathcal{V}, \mathcal{H})$ coincides with the group considered in [AN]. We have the familiar expression

$$
\begin{aligned}
& 0 \longrightarrow H^{1}\left(\mathcal{D}, \underline{\mathbb{k}^{\times}}\right) \stackrel{\text { res }}{\longrightarrow} H^{1}\left(\mathcal{H}, \underline{\mathbb{k}^{\times}}\right) \oplus H^{1}\left(\mathcal{V}, \underline{\mathbb{k}^{\times}}\right) \longrightarrow \operatorname{Aut}(\mathbb{k} \mathcal{T}) \\
& \longrightarrow H^{2}\left(\mathcal{D}, \underline{\mathbb{k}^{\times}}\right) \stackrel{\text { res }}{\longrightarrow} H^{2}\left(\mathcal{H}, \underline{\mathbb{k}^{\times}}\right) \oplus H^{2}\left(\mathcal{V}, \underline{\mathbb{k}^{\times}}\right) \longrightarrow \operatorname{Opext}(\mathcal{V}, \mathcal{H}) \\
& \longrightarrow H^{3}\left(\mathcal{D}, \underline{\mathbb{K}^{\times}}\right) \stackrel{\text { res }}{\longrightarrow} H^{3}\left(\mathcal{H}, \underline{\mathbb{k}^{\times}}\right) \oplus H^{3}\left(\mathcal{V}, \underline{\mathbb{k}^{\times}}\right) \longrightarrow \cdots
\end{aligned}
$$

Notice that, if $(\sigma, \tau) \in Z^{2}\left(\operatorname{Tot} \mathcal{A}^{\bullet}, \bullet\left(\underline{\mathbb{k}^{\times}}\right)\right)$, then it follows from equation (2.6) that

$$
\begin{aligned}
& \sigma\left(\left(\operatorname{id}_{\mathfrak{s}(g)}, g\right),\left(\operatorname{id}_{\mathfrak{s}(h)}, h\right)\right)=1, \quad \\
& \tau\left(\left(x, \operatorname{id}_{\mathfrak{e}(x)}\right),\left(y, \operatorname{id}_{\mathfrak{e}(y)}\right)\right)=1, \quad x, y \in \mathcal{H} .
\end{aligned}
$$

\subsection{Weak Hopf algebras arising from matched pairs of group- oids}

Here we recall the construction of a weak Hopf algebra- actually a face algebra in the sense of Hayashi $[\mathrm{H}]$ - starting from a matched pair of groupoids and an element of the corresponding Opext, given in [AN]. This motivates the discussion in this section as well as the computations performed in the next one.

$$
\begin{aligned}
& \mathcal{B} \rightrightarrows \mathcal{H} \\
& \mathcal{V} \rightrightarrows \mathcal{P}
\end{aligned}
$$

the vacant double groupoid associated to $(\mathcal{H}, \mathcal{V})$. Let $(\sigma, \tau) \in Z^{2}\left(\operatorname{Tot} \mathcal{A}^{\bullet \bullet \bullet}\right.$ $\left.\left(\underline{\mathbb{k}^{\times}}\right)\right)$. Let $\mathbb{k}_{\sigma}^{\tau} \mathcal{T}$ be the vector space with basis $\mathcal{B}$ and multiplication and comultiplication defined on the basis $\mathcal{B}$, respectively by 
- $A . B=\sigma(A, B) \underset{B}{A}$, if $\frac{A}{B}$, and 0 otherwise.

- $\Delta(A)=\sum \tau(B, C) B \otimes C$, where the sum is over all pairs $(B, C)$ with $B \mid C$ and $A=B C$.

TheOREM 2.6. ([AN, Th. 3.8]) (i) $\mathbb{k}_{\sigma}^{\tau} \mathcal{T}$ is a weak Hopf algebra with antipode defined by $\mathcal{S}(A)=\tau\left(A, A^{h}\right)^{-1} \sigma\left(A^{-1}, A^{h}\right)^{-1} A^{-1}, A \in \mathcal{B}$. The source and target subalgebras are commutative of dimension $|\mathcal{P}|$, thus $\mathbb{k}_{\sigma}^{\tau} \mathcal{T}$ is actually a face algebra.

(ii) Let $(\nu, \eta)$ be another normalized 2 -cocycle on $\mathcal{T}$ with values in $\mathbb{k}^{\times}$. Let $\psi: \mathcal{T} \rightarrow \mathbb{k}^{\times}$be a map and let $\Psi: \mathbb{k}_{\sigma}^{\tau} \mathcal{T} \rightarrow \mathbb{k}_{\nu}^{\eta} \mathcal{T}$ be the linear map given by $\Psi(B)=\psi(B) B, B \in \mathcal{B}$. Then $\Psi$ is an isomorphism of quantum groupoids if and only if

$$
\begin{aligned}
& \psi\left(\begin{array}{c}
A \\
B
\end{array}\right) \sigma(A, B)=\psi(A) \psi(B) \nu(A, B), \\
& \text { for all } A, B \in \mathcal{B} \text { such that } \frac{A}{B} ; \\
& \psi(C D) \eta(C, D)=\psi(C) \psi(D) \tau(C, D), \\
& \text { for all } C, D \in \mathcal{B} \text { such that } C \mid D .
\end{aligned}
$$

The category $\operatorname{Rep} \mathbb{k}_{\sigma}^{\tau} \mathcal{T}$ of finite-dimensional representations of the weak Hopf algebra $\mathbb{k}_{\sigma}^{\tau} \mathcal{T}$ has a structure of a $\mathbb{k}$-linear rigid monoidal category $[\mathrm{NV}]$. Recall from [ENO] the definition of a multifusion category: this is a semisimple $\mathbb{k}$-linear rigid tensor category with finitely many isoclasses of simple objects and finite dimensional hom-spaces. A multifusion category is called a fusion category when the unit object is simple.

Proposition 2.7. ([AN, Prop. 3.11]) Keep the notation above. The category $\operatorname{Rep} \mathbb{k}_{\sigma}^{\tau} \mathcal{T}$ of finite dimensional $\mathbb{k}_{\sigma}^{\tau} \mathcal{T}$-modules is a multifusion category. It is fusion if and only if $\mathcal{V} \rightrightarrows \mathcal{P}$ is connected.

\section{§3. Matched pairs of groupoids with connected vertical groupoid}

Let $\mathcal{D} \rightrightarrows \mathcal{P}$ be a connected groupoid. We fix $O \in \mathcal{P}$ and $\tau_{P} \in \mathcal{D}(O, P)$, $P \in \mathcal{P}, \tau_{O}=\operatorname{id}_{O}$. We denote by $D$ the group $\mathcal{D}(O)$; thus $\mathcal{D} \simeq D \times \mathcal{P}^{2}$, with isomorphism given by

$$
\mathcal{D}(P, Q) \ni x \longmapsto\left(\tau_{P} x \tau_{Q}^{-1},(P, Q)\right) .
$$


In other words, we pull back $x$ to an arrow from $O$ to $O$ via the $\tau$ 's:

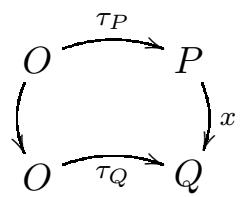

Different choices of families $\tau_{P} \in \mathcal{D}(O, P), P \in \mathcal{P}$, just amount to different isomorphisms of groupoids $\mathcal{D} \simeq D \times \mathcal{P}^{2}$.

\subsection{Structure of exact factorizations}

Let us fix an equivalence relation $\approx_{H}$ on $\mathcal{P}$ and a section $\sigma: \mathcal{P} / \approx_{H} \rightarrow \mathcal{P}$ of the canonical projection. Then there is a bijection between

(a) the set of wide subgroupoids $\mathcal{H}$ of $\mathcal{D}$ with equivalence relation $\approx_{H}$, and

(b) the set of collections $\left(\left(H_{\mathfrak{U}}\right)_{\mathfrak{U} \in \mathcal{P} / \approx_{H}},\left(\overline{\lambda_{P}}\right)_{P \in \mathcal{P}}\right)$, where $H_{\mathfrak{U}}$ is a subgroup of $D, \mathfrak{U} \in \mathcal{P} / \approx_{H}$, and $\overline{\lambda_{P}} \in H_{\mathfrak{U}} \backslash D, P \in \mathfrak{U}$, with $\lambda_{\sigma(\mathfrak{U})} \in H_{\mathfrak{U}}$ for any $\mathfrak{U} \in \mathcal{P} / \approx_{H}$.

Namely, from (a) to (b), if $\mathfrak{U} \in \mathcal{P} / \approx_{H}$ and $P \in \mathfrak{U}$, then we choose $g_{P} \in$ $\mathcal{H}(\sigma(\mathfrak{U}), P)$ and set

$$
H_{\mathfrak{U}}=\tau_{\sigma(\mathfrak{U})} \mathcal{H}(\sigma(\mathfrak{U})) \tau_{\sigma(\mathfrak{U})}^{-1}, \quad \lambda_{P}=\tau_{\sigma(\mathfrak{U})} g_{P} \tau_{P}^{-1} .
$$

The choice of $g_{P}$ does not affect the class of $\lambda_{P}$ in $H_{\mathfrak{U}} \backslash D$. By definition, $\lambda_{\sigma(\mathfrak{U})} \in H_{\mathfrak{U}}$.

Conversely, if a collection $\left(\left(H_{\mathfrak{U}}\right)_{\mathfrak{U} \in \mathcal{P} / \approx_{H}},\left(\overline{\lambda_{P}}\right)_{P \in \mathcal{P}}\right)$ satisfies the above conditions then the wide subgroupoid $\mathcal{H}$ that this collection determines is given by

$$
\mathcal{H}(P, Q)= \begin{cases}\tau_{P}^{-1} \lambda_{P}^{-1} H_{\mathfrak{U}} \lambda_{Q} \tau_{Q}, & \text { if } P \approx_{H} Q, P, Q \in \mathfrak{U} ; \\ \emptyset & \text { if } P \approx_{H} Q .\end{cases}
$$

In other words, $c f$. the equality $\lambda_{P} \tau_{P}=\tau_{\sigma(\mathfrak{U})} g_{P}$, arrows $x$ in $\mathcal{H}$ from $P$ to $Q$ correspond to arrows $\widetilde{x} \in H_{\mathfrak{U}}$ as in this commutative diagram:

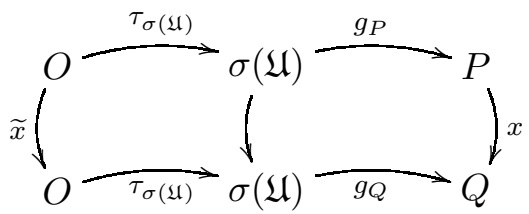


By abuse of notation, we shall say that $\mathcal{H}$ is associated to $\left(\left(H_{\mathfrak{U}}\right)_{\mathfrak{U} \in \mathcal{P} / \approx_{H}}\right.$, $\left.\left(\overline{\lambda_{P}}\right)_{P \in \mathcal{P}}\right)$.

We reformulate the description of exact factorizations of a connected groupoids given in [AN, Th. 2.15] in terms of the preceding discussion. Let us fix equivalence relations $\approx_{V}$ and $\approx_{H}$ on $\mathcal{P}$ and sections $\rho: \mathcal{P} / \approx_{V} \rightarrow \mathcal{P}$, $\sigma: \mathcal{P} / \approx_{H} \rightarrow \mathcal{P}$ of the canonical projections.

THEOREM 3.1. Let $\mathcal{H}$ and $\mathcal{V}$ be wide subgroupoids of $\mathcal{D}$ associated respectively to collections $\left(\left(H_{\mathfrak{U}}\right)_{\mathfrak{U} \in \mathcal{P} / \approx_{H}},\left(\lambda_{P}\right)_{P \in \mathcal{P}}\right),\left(\left(V_{\mathfrak{R}}\right)_{\mathfrak{R} \in \mathcal{P} / \approx_{V}},\left(\mu_{P}\right)_{P \in \mathcal{P}}\right)$ as explained above. Then the following are equivalent:

(i) $\mathcal{D}=\mathcal{V H}$ is an exact factorization.

(ii) $(\mathcal{H}, \mathcal{V})$ is a matched pair of groupoids and $\mathcal{D} \simeq \mathcal{V} \bowtie \mathcal{H}$.

(iii) The following conditions hold:

$$
\begin{aligned}
& \text { (3.1) } \quad D=\coprod_{R \in \mathfrak{U} \cap \mathfrak{R}} V_{\mathfrak{R}} \mu_{R} \lambda_{R}^{-1} H_{\mathfrak{U}}, \quad \text { for all } \mathfrak{U} \in \mathcal{P} / \approx_{H}, \mathfrak{R} \in \mathcal{P} / \approx_{V} ; \\
& \text { (3.2) } \mu_{P}^{-1} V_{\mathfrak{R}} \mu_{P} \cap \lambda_{P}^{-1} H_{\mathfrak{U}} \lambda_{P}=\{1\}, \\
& \text { for all } \mathfrak{U} \in \mathcal{P} / \approx_{H}, \mathfrak{R} \in \mathcal{P} / \approx_{V}, P \in \mathfrak{U} \cap \mathfrak{R} .
\end{aligned}
$$

In the rest of this section we shall study matched pairs of groupoids whose vertical groupoid is connected. These are exactly those such that the tensor category $\operatorname{Rep} \mathbb{k}_{\sigma}^{\tau} \mathcal{T}$ is fusion, see Subsection 2.3.

We fix a vertical subgroupoid $\mathcal{V}$ of $\mathcal{D}$ determined by a subgroup $V$ of $D$ and $\overline{\mu_{P}} \in V \backslash D, P \in \mathcal{P}$. Without loss of generality, we can assume that $\mu_{P}=1$ for all $P \in \mathcal{P}$; just change the family $\left(\tau_{P}\right)$ by $\left(\mu_{P} \tau_{P}\right)$.

We shall say that $\mathcal{H}$ is an exact factor of the subgroupoid $\mathcal{V}$ of $\mathcal{D}$ if $\mathcal{D}=\mathcal{V} \mathcal{H}$ is an exact factorization.

Corollary 3.2. Let $\mathcal{H}$ be a wide subgroupoid of $\mathcal{D}$ associated to $\left(\left(H_{\mathfrak{U}}\right)_{\mathfrak{U} \in \mathcal{P} / \approx_{H}},\left(\lambda_{P}\right)_{P \in \mathcal{P}}\right)$. Then the following are equivalent:

(i) $\mathcal{H}$ is an exact factor of $\mathcal{V}$.

(ii) The following conditions hold:

$$
\begin{aligned}
& D=\coprod_{R \in \mathfrak{U}} V \lambda_{R}^{-1} H_{\mathfrak{U}}, \quad \text { for all } \mathfrak{U} \in \mathcal{P} / \approx_{H} ; \\
& V \cap g H_{\mathfrak{U}} g^{-1}=\{1\}, \quad \text { for all } g \in D .
\end{aligned}
$$


Proof. Condition (3.2) reads now $V \cap \lambda_{P}^{-1} H_{\mathfrak{U}} \lambda_{P}=\{1\}$, for all $\mathfrak{U} \in$ $\mathcal{P} / \approx_{H}, P \in s$. In presence of (3.3), this is equivalent to (3.4).

Summary 3.3. To construct an explicit example of an exact factorization $\mathcal{D}=\mathcal{V} \mathcal{H}$ with $\mathcal{V}$ connected, we need:

- A finite group $D$, a subgroup $V$ of $D$ and a finite non-empty set $\mathcal{P}$. We fix $O \in \mathcal{P}$ and define $\mathcal{D}$ and $\mathcal{V}$ as explained above.

- An equivalence relation $\approx_{H}$ in $\mathcal{P}$.

- A family $\left(H_{\mathfrak{U}}\right)_{\mathfrak{U} \in \mathcal{P} / \approx_{H}}$ of subgroups of $D$ such that

(a) $V$ intersects trivially all conjugates of $H_{\mathfrak{U}}$ for all $\mathfrak{U} \in \mathcal{P} / \approx_{H}$.

(b) There are bijections $\varphi_{\mathfrak{U}}: V \backslash D / H_{\mathfrak{U}} \simeq \mathfrak{U}$ for all $\mathfrak{U} \in \mathcal{P} / \approx_{H}$.

We denote $\sigma(\mathfrak{U})=\varphi_{\mathfrak{U}}\left(V H_{\mathfrak{U}}\right) \in \mathfrak{U}$.

- A section $\zeta: \mathfrak{U} \rightarrow D$ of the canonical projection $D \rightarrow V \backslash D / H_{\mathfrak{U}}$ composed with $\varphi_{\mathfrak{U}}$, such that $\zeta_{\sigma(\mathfrak{U})} \in H_{\mathfrak{U}}$.

We set $\lambda_{P}=\zeta_{P}^{-1}$ for any $P \in \mathcal{P}$; and clearly $\lambda_{\sigma(\mathfrak{U})}=\zeta_{\sigma(\mathfrak{U})}^{-1} \in H_{\mathfrak{U}}$. Then $\mathcal{H}$ is the wide subgroupoid associated to $\left(\left(H_{\mathfrak{U}}\right)_{\mathfrak{U} \in \mathcal{P} / \approx_{H}},\left(\lambda_{P}\right)_{P \in \mathcal{P}}\right)$; it is an exact factor of $\mathcal{V}$ by (a) and (b).

Remark 3.4. If $D$ is a finite group, $V$ and $H$ are subgroups of $D$ with double coset decomposition $D=\coprod_{P \in \mathfrak{U}} V \zeta_{P} H$, then $[D: H]=\sum_{P \in \mathfrak{U}}[V$ : $\left.V \cap \zeta_{P} H \zeta_{P}^{-1}\right]$; if in addition $V \cap g H g^{-1}=\{1\}$ for all $g \in D$ then $|D|=$ $\# \mathfrak{U}|V||H|$. See [AM, p. 76].

We shall next analyze explicit examples of exact factors $\mathcal{H}$ of $\mathcal{V}$ according to the equivalence relation $\approx_{H}$.

\subsection{Case 1 . The equivalence relation $\approx_{H}$ is totally discon- nected}

Here $\approx_{H}$ is the identity relation: if $P \approx_{H} Q$ then $P=Q$ for any $P, Q \in \mathcal{P}$. Wide subgroupoids $\mathcal{H}$ with this equivalence relation correspond to families $\left(H_{P}\right)_{P \in \mathcal{P}}$ of subgroups of $D$.

Summary 3.5. Let $\mathcal{H}$ be as above. Then the following statements are equivalent:

(i) $\mathcal{D}=\mathcal{V H}$ is an exact factorization. 
(ii) $D=V H_{P}$ is an exact factorization for any $P \in \mathcal{P}$.

To construct an explicit example of an exact factorization $\mathcal{D}=\mathcal{V H}$ with $\mathcal{V}$ connected and $\approx_{H}=\mathrm{id}$, we need a finite group $D$, a $\operatorname{subgroup} V$ and a family $\left(H_{P}\right)_{P \in \mathcal{P}}$ of subgroups of $D$ (thus the index set $\mathcal{P}$ is the basis of the groupoid) such that $H_{P}$ is an exact factor of $V$ for any $P \in \mathcal{P}$.

Given a finite group $D$ and a subgroup $V$, we are thus faced to the problem of finding all exact factors $H$ of $V$. We observe that:

(0) Any conjugate of an exact factor of $V$ is again an exact factor of $V$.

(1) There exist a finite group $D$, a subgroup $V$ and exact factors $H$ and $H^{\prime}$ such that $H \nsubseteq H^{\prime}$. For instance, $D=\mathbb{S}_{4}, V=\mathbb{S}_{3}$ (the subgroup that fixes 4$), H=\langle(1234)\rangle \simeq \mathbb{Z} /(4), H^{\prime}=\langle(24)(13),(34)(12)\rangle \simeq \mathbb{Z} /(2) \oplus \mathbb{Z} /(2)$.

(2) There exist a finite group $D$, a subgroup $V$ and exact factors $H$ and $H^{\prime}$ with $H \simeq H^{\prime}$ but $H$ not conjugate to $H^{\prime}$. For instance, $D=\mathbb{S}_{n}, n \geq 6$, $V=A_{n}, H=\langle(12)\rangle, H^{\prime}=\langle(12)(34)(56)\rangle$.

(3) (Schur-Zassenhaus Theorem) If $D$ is a finite group and $V \triangleleft D$ is a normal subgroup such that $(|V|,[D: V])=1$, then $V$ admits exact factors, which are all conjugate. The known proof of the conjugacy of the exact factors relies on the Feit-Thompson Theorem (any group of odd order is solvable).

(4) The list of all exact factorizations of $\mathbb{S}_{n}$ and $\mathbb{A}_{n}$ is given in [WW].

Let $\mathcal{D}=\mathcal{V H}$ be an exact factorization with $\mathcal{V}$ connected and $\approx_{H}=$ id. Then, by Proposition 1.10 (i), the Kac exact sequence (2.7) has the form (3.5)

$$
\begin{aligned}
0 \longrightarrow H^{1}\left(D, \mathbb{k}^{\times}\right) \stackrel{\text { res }}{\longrightarrow} \bigoplus_{P \in \mathcal{P}} H^{1}\left(H_{P}, \mathbb{k}^{\times}\right) \oplus H^{1}\left(V, \mathbb{k}^{\times}\right) \longrightarrow \operatorname{Aut}(\mathbb{k} \mathcal{T}) \\
\longrightarrow H^{2}\left(D, \mathbb{k}^{\times}\right) \stackrel{\text { res }}{\longrightarrow} \bigoplus_{P \in \mathcal{P}} H^{2}\left(H_{P}, \mathbb{k}^{\times}\right) \oplus H^{2}\left(V, \mathbb{k}^{\times}\right) \longrightarrow \operatorname{Opext}(\mathcal{V}, \mathcal{H}) \\
\longrightarrow H^{3}\left(D, \mathbb{k}^{\times}\right) \stackrel{\text { res }}{\longrightarrow} \bigoplus_{P \in \mathcal{P}} H^{3}\left(H_{P}, \mathbb{k}^{\times}\right) \oplus H^{3}\left(V, \mathbb{k}^{\times}\right) \longrightarrow \cdots
\end{aligned}
$$

ExAmple 3.6. Let $m \in \mathbb{N}, m \geq 5$. Let $D:=\mathbb{S}_{m}, V:=C_{m}=$ $\langle(1 \ldots m)\rangle, \mathcal{P}:=\{1 \ldots m\}, H_{i}:=\mathbb{S}_{m}^{(i)}:=\left\{\sigma \in \mathbb{S}_{m}: \sigma(i)=i\right\}, 1 \leq i \leq m$. The groups $\mathbb{S}_{m}^{(i)}$ are conjugate to each other, indeed if $\tau_{i j}=(i j)$ then $\tau_{i j} \mathbb{S}_{m}^{(i)} \tau_{i j}^{-1}=\mathbb{S}_{m}^{(j)}$. We have exact factorizations $\mathbb{S}_{m}=C_{m} \mathbb{S}_{m}^{(i)}$ for any $1 \leq i \leq m$, hence we have an exact factorization of groupoids $\mathcal{D}=\mathcal{V H}$. 
Assume now that $\mathbb{k}=\mathbb{C}$. We rely on calculations done in [M2]. We claim that $\operatorname{Opext}(\mathcal{V}, \mathcal{H})=\mathbb{Z}_{2}^{m-1}$. It is known that $H^{2}\left(C_{m}, \mathbb{k}^{\times}\right)=0$ that $H^{2}\left(\mathbb{S}_{m}, \mathbb{k}^{\times}\right)=\mathbb{Z}_{2}$; that the restriction map res : $H^{2}\left(\mathbb{S}_{m}, \mathbb{k}^{\times}\right) \rightarrow$ $H^{2}\left(\mathbb{S}_{m-1}, \mathbb{k}^{\times}\right)$is bijective [M2, p. 579]; and that res : $H^{3}\left(\mathbb{S}_{m}, \mathbb{k}^{\times}\right) \rightarrow$ $H^{3}\left(\mathbb{S}_{m-1}, \mathbb{k}^{\times}\right) \oplus H^{3}\left(C_{m}, \mathbb{k}^{\times}\right)$is an injective map. Hence the Kac exact sequence $(3.5)$ gives $0 \rightarrow \mathbb{Z}_{2} \rightarrow \mathbb{Z}_{2}^{m} \rightarrow \operatorname{Opext}(\mathcal{V}, \mathcal{H}) \rightarrow 0$ and the claim follows.

\subsection{Case 2. The equivalence relation $\approx_{H}$ is connected}

Here both $\approx_{V}$ and $\approx_{H}$ are connected. A wide subgroupoid $\mathcal{H}$ in this case corresponds to a subgroup $H$ of $D$ and elements $\left(\lambda_{P}\right)_{P \in \mathcal{P}} \in H \backslash D$, such that $\lambda_{O} \in H$.

Summary 3.7. Let $\mathcal{H}$ be a groupoid as above. Then the following statements are equivalent.

(i) $\mathcal{D}=\mathcal{V H}$ is an exact factorization.

(ii) $D=\coprod_{r \in \mathcal{P}} V \lambda_{r}^{-1} H$ (thus $\#(V \backslash D / H)=\# \mathcal{P}$ ) and $V \cap g H g^{-1}=\{1\}$ for any $g \in D$.

To construct an explicit example of an exact factorization $\mathcal{D}=\mathcal{V H}$ with $\mathcal{V}$ and $\mathcal{H}$ connected, we need a finite group $D$, two subgroups $V$ and $H$ such that $V \cap g H g^{-1}=\{1\}$ for any $g \in D$, and a section $\zeta: V \backslash D / H \rightarrow D$ of the canonical projection $D \rightarrow V \backslash D / H$ such that $\zeta(V H) \in H$. The base of the groupoids $\mathcal{D}, \mathcal{V}$ and $\mathcal{H}$ is $\mathcal{P}:=V \backslash D / H$, and $\zeta_{P}=\lambda_{P}^{-1}$ for any $P \in \mathcal{P}$.

Given a finite group $D$ and a subgroup $V$, we have to find subgroups $H$ of $D$ such that $V$ intersects trivially all conjugates of $H$. We observe that:

(1) If the orders $|V|,|H|$ are relatively prime then this condition is automatically fulfilled.

(2) If $V$ admits an exact factor $K$ and $H$ is any subgroup of $K$ then $V$ intersects trivially all conjugates of $H$.

Let $\mathcal{D}=\mathcal{V H}$ be an exact factorization with $\mathcal{V}$ and $\mathcal{H}$ connected. Then, by Proposition 1.10 (i), the Kac exact sequence (2.7) has the form

$$
\begin{aligned}
& 0 \longrightarrow H^{1}\left(D, \mathbb{k}^{\times}\right) \stackrel{\text { res }}{\longrightarrow} H^{1}\left(H, \mathbb{k}^{\times}\right) \oplus H^{1}\left(V, \mathbb{k}^{\times}\right) \longrightarrow \operatorname{Aut}(\mathbb{k} \mathcal{T}) \\
& \longrightarrow H^{2}\left(D, \mathbb{k}^{\times}\right) \stackrel{\text { res }}{\longrightarrow} H^{2}\left(H, \mathbb{k}^{\times}\right) \oplus H^{2}\left(V, \mathbb{k}^{\times}\right) \longrightarrow \operatorname{Opext}(\mathcal{V}, \mathcal{H}) \\
& \longrightarrow H^{3}\left(D, \mathbb{k}^{\times}\right) \stackrel{\text { res }}{\longrightarrow} H^{3}\left(H, \mathbb{k}^{\times}\right) \oplus H^{3}\left(V, \mathbb{k}^{\times}\right) \longrightarrow \cdots
\end{aligned}
$$


EXAmPle 3.8. Let $m, k, r \in \mathbb{N}$ be such that $k, r \leq m$ and $k>m-r$. Let $X \subseteq\{1, \ldots, m\}$ be a subset of cardinal $r$. Let $D:=\mathbb{S}_{m}, V:=C_{k}=$ $\langle(12 \ldots k)\rangle, H:=\mathbb{S}_{m}^{X}:=\left\{\sigma \in \mathbb{S}_{m}: \sigma(x)=x\right.$ for all $\left.x \in X\right\} \simeq \mathbb{S}_{m-r}$. Since $\sigma \mathbb{S}_{m}^{X} \sigma^{-1}=\mathbb{S}_{m}^{\sigma(X)}$ for any $\sigma \in \mathbb{S}_{m}$, then $C_{k} \cap \sigma \mathbb{S}_{m}^{X} \sigma^{-1}=\{e\}$ for any $\sigma \in \mathbb{S}_{m}$. In this example $\# \mathcal{P}=n(n-1) \cdots(n-r+1) / k$.

Let us assume that we are in conditions of Summary 3.7. The set $V H \subseteq$ $D$ is a subgroup of $D$ if and only if $V H=H V$. If this is the case, we have an exact factorization that allows us to consider the group $\operatorname{Opext}(V, H)$. The following example shows that the isomorphism $\operatorname{Opext}(V, H) \simeq \operatorname{Opext}(\mathcal{V}, \mathcal{H})$ does not necessarily hold.

ExAmple 3.9. In this example $\mathbb{k}=\mathbb{C}$. Let $p, q, n \in \mathbb{N}$, where $p$ and $q$ are relatively prime, and set $m=p q n$. Let $D:=\mathbb{Z} /(m), V:=\mathbb{Z} /(p), H:=$ $\mathbb{Z} /(q)$. Let $\mathcal{D}, \mathcal{V}$ and $\mathcal{H}$ the corresponding connected groupoids associated to $D, V$ and $H$ respectively, with $\mathcal{P}=V \backslash D / H$ of cardinal $n$.

We claim that $\operatorname{Opext}(\mathcal{V}, \mathcal{H}) \simeq \mathbb{Z} /(n)$. Indeed, since $H^{2}\left(\mathbb{Z} /(p), \mathbb{C}^{\times}\right)=$ $H^{2}\left(\mathbb{Z} /(q), \mathbb{C}^{\times}\right)=0$ then $\operatorname{Opext}(\mathcal{V}, \mathcal{H}) \simeq \operatorname{Ker}\left(\right.$ res $: H^{3}\left(\mathbb{Z} /(m), \mathbb{C}^{\times}\right) \rightarrow$ $\left.H^{3}\left(\mathbb{Z} /(p), \mathbb{C}^{\times}\right) \oplus H^{3}\left(\mathbb{Z} /(q), \mathbb{C}^{\times}\right)\right)$by $(3.6)$. Now, it is known that $H^{3}(\mathbb{Z} /(r)$, $\left.\mathbb{C}^{\times}\right) \simeq \mathbb{Z} /(r)$ for any $r \in \mathbb{N}[\mathrm{AM}$, p. 61$]$. And it is not difficult to see that the restriction map res : $\mathbb{Z} /(m) \rightarrow \mathbb{Z} /(p) \oplus \mathbb{Z} /(q)$ via these isomorphisms is the canonical projection, and thus $\operatorname{Opext}(\mathcal{V}, \mathcal{H}) \simeq \mathbb{Z} /(n) \not \operatorname{Opext}(V, H)$ unless $n=1$.

\subsection{Case 3. Equivalence relations $\approx_{H}$ with two classes}

We assume here that $\approx_{H}$ has two equivalence classes: $\{O\}$, and $\mathcal{P}-\{O\}$. We fix $\widetilde{O} \in \mathcal{P}-\{O\}$. A wide subgroupoid $\mathcal{H}$ in this case corresponds to a pair of subgroups $H_{1}, H_{2}$ and a family $\left(\lambda_{P}\right)_{P \in \mathcal{P}-\{O\}}$ in $D$ such that $\lambda_{\widetilde{O}} \in H_{2}$.

Summary 3.10. Let $\mathcal{H}$ be a groupoid as above. The following statements are equivalent.

(i) $\mathcal{D}=\mathcal{V H}$ is an exact factorization,

(ii) (a) $D=V H_{1}$ is an exact factorization,

(b) $D=\coprod_{P \in \mathcal{P}-\{O\}} V \lambda_{P}^{-1} H_{2}$ and $V \cap g H_{2} g^{-1}=\{1\}$ for any $g \in D$. 
By Proposition 1.10 (i) the Kac exact sequence has the form $(3.7)$

$$
\begin{aligned}
0 \longrightarrow H^{1}\left(D, \mathbb{k}^{\times}\right) \stackrel{\text { res }}{\longrightarrow} H^{1}\left(H_{1}, \mathbb{k}^{\times}\right) \oplus H^{1}\left(H_{2}, \mathbb{k}^{\times}\right) \oplus H^{1}\left(V, \mathbb{k}^{\times}\right) \longrightarrow \operatorname{Aut}(\mathbb{k} \mathcal{T}) \\
\longrightarrow H^{2}\left(D, \mathbb{k}^{\times}\right) \stackrel{\text { res }}{\longrightarrow} H^{2}\left(H_{1}, \mathbb{k}^{\times}\right) \oplus H^{2}\left(H_{2}, \mathbb{k}^{\times}\right) \oplus H^{2}\left(V, \mathbb{k}^{\times}\right) \longrightarrow \operatorname{Opext}(\mathcal{V}, \mathcal{H}) \\
\longrightarrow H^{3}\left(D, \mathbb{k}^{\times}\right) \stackrel{\text { res }}{\longrightarrow} H^{3}\left(H_{1}, \mathbb{k}^{\times}\right) \oplus H^{3}\left(H_{2}, \mathbb{k}^{\times}\right) \oplus H^{3}\left(V, \mathbb{k}^{\times}\right) \longrightarrow \cdots .
\end{aligned}
$$

Examples in this case are obtained combining the examples in the previous cases. We note a general way of obtaining collections as in Summary 3.10. If $D=V H_{1}$ is an exact factorization and $H_{2}$ is a subgroup of $H_{1}$, then $V \cap g H_{2} g^{-1}=\{1\}$ for any $g \in D$. Let us discuss an explicit example.

ExAMPLE 3.11. Let $H_{2}$ be any group of order $n$, considered as a subgroup of $\mathbb{S}_{n}$ via, say, the left regular action on itself. Set $D=\mathbb{S}_{n+1}$, $V=\langle(12 \ldots n+1)\rangle \simeq C_{n+1}, H_{1}=\mathbb{S}_{n}$, an exact factor of $V$. By (3.7), we have

$$
\operatorname{Opext}(\mathcal{V}, \mathcal{H}) \cong H^{2}\left(H_{2}, \mathbb{k}^{\times}\right) / \operatorname{Im}\left(\operatorname{res}: H^{2}\left(\mathbb{S}_{n+1}, \mathbb{k}^{\times}\right) \rightarrow H^{2}\left(H_{2}, \mathbb{k}^{\times}\right)\right) .
$$

There are also examples which are not of this form.

EXAMPLE 3.12. Let $n \in \mathbb{N}$ such that $n=r s$ with $r, s \neq 1, r, s \in \mathbb{N}$. Let $D=\mathbb{S}_{n}, V=\mathbb{S}_{n-1}, H_{1}=\langle(1, \ldots, n)\rangle$ and $H_{2}=\langle\sigma\rangle$, where $\sigma=$ $(1 \ldots r)(r+1 \ldots 2 r) \cdots(r s-r+1 \ldots r s)$. Then $H_{1}$ is an exact factor of $V, V$ intersects trivially any conjugate of $H_{2}$ and $\sigma \notin H_{1}$. In this case $\# \mathcal{P}=s+1$.

\section{REFERENCES}

[AN] N. Andruskiewitsch and S. Natale, Double categories and quantum groupoids, Publ. Mat. Urug., 10 (2005), 11-51.

[AM] A. Adem and R. J. Milgram, Cohomology of Finite Groups, Springer-Verlag, 1994.

[BaSV] S. Baaj, G. Skandalis and S. Vaes, Measurable Kac cohomology for bicrossed products, Trans. Amer. Math. Soc., 357 (2005), 1497-1524.

[BNS] G. Böhm, F. Nill and K. Szlachányi, Weak Hopf algebras I. Integral theory and $C^{*}$-structure, J. Algebra, 221 (1999), 385-438.

[BS] G. Böhm and K. Szlachányi, A coassociative $C^{*}$-quantum group with nonintegral dimensions, Lett. Math. Phys., 35 (1996), 437-456.

[BW] T. Brzezinski and R. Wisbauer, Corings and Comodules, Cambridge University Press, 2003. 
[CE] H. Cartan and S. Eilenberg, Homological Algebra, Princeton University Press, 1956.

[ENO] P. Etingof, D. Nikshych and V. Ostrik, On fusion categories, Annals of Math., 162 (2005), 581-642.

[H] T. Hayashi, A brief introduction to face algebras, New trends in Hopf Algebra Theory, Contemp. Math. 267 (2000), pp. 161-176.

[K] C. Kassel, Quantum groups, Springer-Verlag, 1995.

[Ma] K. Mackenzie, Double Lie algebroides and Second-order Geometry I, Adv. Math., 94 (1992), 180-239.

[M1] A. Masuoka, Hopf algebra extensions and cohomology, Math. Sci. Res. Inst. Publ., 43 (2002), 167-209.

[M2] A. Masuoka, Calculations of Some Groups of Hopf Algebra Extensions, J. Algebra, 191 (1997), 568-588.

[NV] D. Nikshych and L. Vainerman, Finite quantum groupoids and their applications, Recent developments in Hopf algebra theory, Math. Sci. Res. Inst. Publ. 43 (2002), pp. 211-262.

[R] J. Renault, A groupoid approach to $C^{*}$-algebras, Lect. Notes Math. 793, Springer-Verlag, Berlin, 1980.

[W] C. Weibel, An introduction to homological algebra, Cambridge University Press, 1994.

[WW] J. Wiegold and A. G. Williamson, The Factorizations of the Alternating and Symmetric Groups, Math. Z., 175 (1980), 171-179.

\author{
Nicolás Andruskiewitsch \\ Facultad de Matemática, Astronomía y Física \\ Universidad Nacional de Córdoba \\ CIEM - CONICET \\ Medina Allende $s / n$ \\ (5000) Ciudad Universitaria, Córdoba \\ Argentina \\ andrus@mate . uncor . edu \\ Juan Martín Mombelli \\ Facultad de Matemática, Astronomía y Física \\ Universidad Nacional de Córdoba \\ CIEM - CONICET \\ Medina Allende $s / n$ \\ (5000) Ciudad Universitaria, Córdoba \\ Argentina \\ mombelli@mate.uncor.edu
}

